\title{
IFI30 as a prognostic biomarker and correlation with immune infiltrates in glioma
}

\author{
Wei Jiang $^{1 \#}$, Feifei Zheng ${ }^{2 \#}$, Taotao Yao ${ }^{3 \#}$, Fang Gong $^{2}$, Wenjie Zheng ${ }^{4}$, Ninghua Yao ${ }^{5 \wedge}$ \\ ${ }^{1}$ Department of Neurology, the Second People's Hospital of Wuxi, Wuxi, China; ${ }^{2}$ Department of Laboratory Medicine, Affiliated Hospital of \\ Jiangnan University, Wuxi, China; ${ }^{3}$ Rehabilitation Center, the First Affiliated Hospital of Guangzhou University of Traditional Chinese Medicine, \\ Guangzhou, China; ${ }^{4}$ Research Center of Clinical Medicine, Affiliated Hospital of Nantong University, Nantong, China; ${ }^{5}$ Department of Oncology, \\ Affiliated Hospital of Nantong University, Nantong, China \\ Contributions: (I) Conception and design: W Jiang, N Yao; (II) Administrative support: F Gong, W Zheng; (III) Provision of study materials or \\ patients: F Zheng; (IV) Collection and assembly of data: F Zheng, T Yao; (V) Data analysis and interpretation: W Jiang, N Yao; (VI) Manuscript \\ writing: All authors; (VII) Final approval of manuscript: All authors. \\ \#These authors contributed equally to this work. \\ Correspondence to: Dr. Ninghua Yao. Department of Oncology, Affiliated Hospital of Nantong University, Nantong 226001, China. \\ Email: yaonh2009@163.com.
}

Background: Increased evidence indicates that the tumour microenvironment (TME) plays an essential role in the development, treatment and prognosis of glioma. High expression of interferon-gamma-inducible protein 30 (IFI30) is associated with the malignant phenotype, but the effect of IFI30 on the tumour immune microenvironment and its potential role in the carcinogenesis of glioma remain unknown.

Methods: The RNA sequencing (RNA-seq) data of 33 types of human cancer were obtained from The Cancer Genome Atlas (TCGA) Genomic Data Commons (GDC). R software was used to perform analyses, such as the expression of IFI30 in pan-cancer, evaluation of IFI30 as a prognostic biomarker in glioma, the relationship between IFI30 expression and clinical characteristics, and immune checkpoint. TIMER was used to analyse the correlation of IFI30 expression level with immune cell infiltration, and also to conduct survival analysis for immune cells and IFI30 in low grade glioma (LGG). DAVID was used for Gene Ontology (GO) functional annotations and Kyoto Encyclopedia of Genes and Genomes (KEGG) for pathway analysis of the genes similar to IFI30 in glioma. The differentially expressed genes (DEGs) between the high- and low-IFI30 expression groups were determined by DESeq2. Gene set enrichment analysis (GSEA) was then conducted to identify IFI30-related functional significance based on the hallmark gene set.

Results: Dysregulated expression of IFI30 was associated with human cancers. High IFI30 expression was associated with poor overall survival (OS), disease-specific survival (DSS) and progression-free interval (PFI). Univariate and multivariate analyses identified IFI30 as an independent predictor for glioma. Meanwhile, IFI30 overexpression significantly correlated with high-grade tumours, poor OS, and immune infiltration. In addition, IFI30-associated genes significantly enriched the hallmark tumour progression-related clusters and cancer pathways.

Conclusions: IFI30 is a prognostic biomarker correlated with immune infiltrates and acts as an oncogene in glioma.

Keywords: Interferon-gamma-inducible protein 30 (IFI30); glioma; prognosis; tumour immune infiltration

Submitted Sep 24, 2021. Accepted for publication Nov 12, 2021.

doi: 10.21037/atm-21-5569

View this article at: https://dx.doi.org/10.21037/atm-21-5569

$\wedge$ ORCID: 0000-0002-7607-2516. 


\section{Introduction}

Glioma is one of the most malignant tumours in the brain, accounting for around $27 \%$ of all primary brain tumours and is responsible for approximately 13,000 cancer-related deaths in the USA each year $(1,2)$. Among them, glioblastoma multiforme (GBM) accounts for $40-50 \%$ of glioma. The standard treatment is surgery and radiotherapy, which are the most common and extremely fatal brain tumours. Despite advances in surgical resection and chemoradiotherapy, the prognosis of glioma patients remains dismal (3-5). Thus, it is extremely meaningful and urgent to identify more sensitive and specific prognostic biomarkers.

The tumour microenvironment (TME) comprises infiltrating inflammatory cells, blood vessels, tumour cells, tumour stroma and various associated tissue cells (6). Tumours shape the TME by recruiting stromal cells, such as tumourassociated macrophages and regulatory $T$ cells (Tregs), all of which are associated with poor prognosis $(7,8)$. In addition, tumour-derived factors promote the release of nutrients from the TME to promote tumour growth (9). Therefore, increasing evidence indicates that the TME plays an important role in tumour development, treatment and prognosis (10). In addition to cancer cells, the TME also contains many different types of non-cancerous cells, including endothelial cells, immune cells, which protect the brain from inflammation. A number of studies in the past decade have shown that the brain TME is the basic regulator of cancer progression and the therapeutic effect of primary and metastatic brain malignancies. In recent years it has been found that the brain is also connected to the peripheral immune system through the lymphatic vessels in the meninges (11). This finding shed new light on the relationship between the brain's immune microenvironment and neurological diseases, including glioma. Therefore, the focus of glioma research has moved to the tumour immune microenvironment.

Interferon-gamma-inducible protein 30 (IFI30), also known as gamma-interferon-inducible lysosomal thiol reductase (GILT), is involved in major histocompatibility complex (MHC) class I-restricted cross-presentation and MHC class II-restricted antigen processing pathways of adaptive immunity and constitutively expressed in most antigen-presenting cells, including B cells, bone marrowderived dendritic cells, monocytes, and macrophages (12-14). IFI30 is also expressed at increased levels in various cancers such as breast cancer and melanoma $(12,15)$. In addition, IFI30 may be linked to disease progression in prostate cancer (16). Although IFI30 is highly expressed in certain APCs, such as dendritic cells, B cells, and macrophages, and is known as an immune activator that promotes the antigen presentation process, few studies currently estimate the role of IFI30 in tumour cells. Recent studies demonstrated an association between IFI30 expression and the malignant phenotype of glioma (17). However, the effects of IFI30 on the tumour immune microenvironment and its potential role in the carcinogenesis of glioma remain unknown. Although reports have studied the important role of IFI30 in glioma, it has not yet been evaluated whether IFI30 can be used as an independent prognostic factor for patients with glioma. This study analysed the prognostic value of IFI30 in glioma and its relationship with tumour immune infiltration.

In this study, we used The Cancer Genome Atlas (TCGA) pan-cancer data to analyse the expression of IFI30 in tumour tissues and normal tissues. We present the following article in accordance with the REMARK reporting checklist (available at https://dx.doi.org/10.21037/atm-21-5569).

\section{Methods}

\section{TCGA pan-cancer and glioma data acquisition}

The level three RNA sequencing (RNA-seq) data [fragments per kilobase per million (FPKM)] of 33 types of human cancer, including TCGA-low grade glioma (LGG) dataset, TCGAglioblastoma multiforme (GBM) dataset and corresponding clinical information, were obtained from TCGA Genomic Data Commons (GDC) (https://portal.gdc.cancer.gov/). RNAseq data (FPKM values) were then transformed to transcripts per million reads (TPM) and normalised into $\log 2(\mathrm{TPM}+1)$. The study was conducted in accordance with the Declaration of Helsinki (as revised in 2013).

\section{Gene Ontology (GO) annotations and Kyoto Encyclopedia of Genes and Genomes (KEGG) pathways analysis}

Gene Expression Profiling Interactive Analysis (GEPIA) (http://gepia.cancer-pku.cn/), a web-based tool, was used to obtain the top 120 similar genes of IFI30 in LGG and GBM tumours. DAVID (https://david.ncifcrf.gov) was used for the GO functional annotations and KEGG for pathways analysis of the top similar genes.

\section{Differential gene expression and gene set enrichment analysis (GSEA) analysis}

The differentially expressed genes (DEGs) between high- 
and low-IFI30 expression groups were determined by DESeq2 with $\mid \log _{2}[$ fold change $(\mathrm{FC})] \mid>1$ and adjusted $\mathrm{P}$ value $<0.05$ (18). GSEA (https://software.broadinstitute. org/gsea/index.jsp) was then conducted to identify IFI30related functional significance based on the hallmark gene set ("h.all.v7.0.symbols.gmt"). INormalised enrichment score (NES) $\mid>1$, adjusted $\mathrm{P}$ value $<0.05$, and false discovery rate $(\mathrm{FDR})<0.05$ were considered to be statistically significant.

\section{TIMER database analysis}

TIMER (https://cistrome.shinyapps.io/timer/) was used to analyse the correlation of IFI30 expression level with immune cell infiltration level in LGG. $\mathrm{P}$ value $<0.05$ was considered statistically significant. TIMER was also used to conduct survival analysis for immune cells and IFI30 in LGG. Log-rank P value $<0.05$ was regarded as statistically significant.

\section{Additional bioinformatic and statistical analysis}

R software (version 4.1.0, https://www.r-project.org/) was used to perform analyses and plot graphs. Patients' characteristics between groups were compared using the chi-square test and Fisher's exact test. The Wilcoxon's ranksum test was used for unpaired samples, and the Wilcoxon signed-rank test was used for paired samples. Correlations between the levels of IFI30 and immune checkpoints in glioma were also analysed using the Spearman correlation. Survival analysis for IFI30 was carried out and visualised by the R survival package (https://CRAN.R-project.org/ package=survival). Kruskal-Wallis test was used to analyse the IFI30 expression among different clinicopathological features. Visualisation of the results of analyses was performed using the R package ggplot2 (https://ggplot2.org) (19). P value $<0.05$ was considered statistically significant.

\section{Results}

Inspection of IFI30 mRNA expression data of 33 cancer types in the TCGA database

First, we obtained the differential expression of IFI30 in cancer and normal tissue samples from the TCGA database, as shown in Figure 1A. In bladder urothelial carcinoma (BLCA), breast invasive carcinoma (BRCA), cervical squamous cell carcinoma and endocervical adenocarcinoma
(CECS), cholangiocarcinoma (CHOL), esophageal carcinoma (ESCA), GBM, head and neck squamous cell carcinoma (HNSC), kidney chromophobe (KICH), kidney renal clear cell carcinoma (KIRC), kidney renal papillary cell carcinoma (KIRP), liver hepatocellular carcinoma (LIHC), prostate adenocarcinoma (PAD), stomach adenocarcinoma (STAD), thyroid carcinoma (THCA), and uterine corpus endometrial carcinoma (UCEC), IFI30 expression was increased in tumour tissues compared with normal tissues. In lung adenocarcinoma (LUAD), lung squamous cell carcinoma (LUSC), and pancreatic adenocarcinoma (PAAD), IFI30 expression was reduced in tumour tissues compared with normal tissues. There was no difference in the expression of IFI30 in normal and tumour tissues between colon adenocarcinoma (COAD) and rectum adenocarcinoma (READ).

We also used the TCGA database to analyse the expression of IFI30 in LGG, GBM, UCEC, ESCA, BRCA, HNSC, LIHC, and STAD cancers and adjacent normal tissues. We observed that the expression of IFI30 in cancer tissues was higher than in normal tissues (Figure 1B-1I).

\section{Evaluation of IFI30 as a prognostic biomarker in glioma}

A difference of IFI30 expression in glioma (LGG and GBM) tissue and normal brain tissue was observed. We compared overall survival (OS) between the high IFI30 expression group and low IFI30 expression group in TCGA-LGG and TCGA-GBM cohorts. Median OS for the high IFI30 group was 63.8 (46.7-95.8) months and 115.7 (81.1-148.2) months for the low IFI30 group in LGG $(\mathrm{P}<0.001$; Figure $2 A)$. Median OS for the high IFI30 group was $11.2(9-13.1)$ months, and $16(14.9-18.1)$ months for the low IFI30 group in GBM $(\mathrm{P}=0.008$; Figure $2 B)$. Considering the possibility of non-tumour death during follow-up, we analysed the relationship between IFI30 gene expression and disease-specific survival (DSS) in LGG and GBM. Median DSS for the high IFI30 group was 74.5 (52.6-95.8) months, and 119 (81.1-156.5) months for the low IFI30 group in LGG $(\mathrm{P}<0.001$; Figure $2 C)$. Median DSS for the high IFI30 group was 12 (10-13.5) months, and $16.2(15.2-18.3)$ months for the low IFI30 group in GBM $(\mathrm{P}=0.006$; Figure $2 D)$. In addition, we analysed the relationship between IFI30 expression and the progression-free interval (PFI) in LGG and GBM. Median PFI for the high IFI30 group was 31.7 (24.1-43.5) months and $53.5(40.2-75.6)$ months for the low IFI30 group in LGG (P=0.003; Figure 2E). Median PFI for the high IFI30 

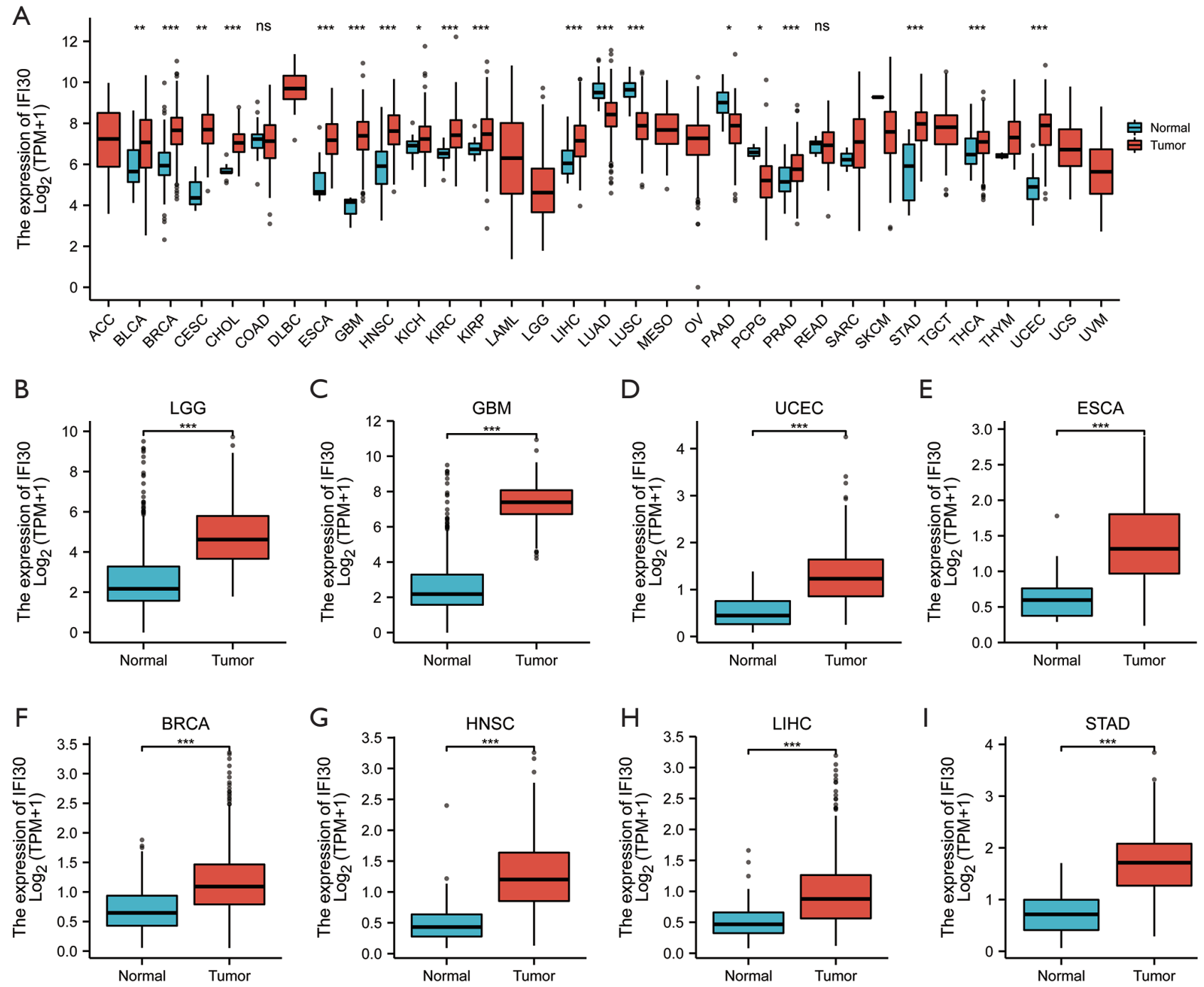

Figure 1 Expression analysis of IFI30 in pan-cancer data. (A) Expression of IFI30 in 33 types of human cancer based on TCGA cancer and normal tissue data. (B-I) Representative IFI30 expression in TCGA-LGG (B), GBM (C), UCEC (D), ESCA (E), BRCA (F), HNSC $(\mathrm{G})$, LIHC $(\mathrm{H})$ and STAD (I) tissues compared with corresponding TCGA normal tissues. *, $\mathrm{P}$ value $\leq 0.05$; **, $\mathrm{P}$ value $\leq 0.01$; ***, $\mathrm{P}$ value $\leq 0.001$; ns, $\mathrm{P}$ value $>0.05$. IFI30, interferon-gamma-inducible protein 30; TCGA, The Cancer Genome Atlas; LGG, low grade glioma; GBM, glioblastoma multiforme; UCEC, uterine corpus endometrial carcinoma; ESCA, esophageal carcinoma; BRCA, breast invasive carcinoma; HNSC, head and neck squamous cell carcinoma; LIHC, liver hepatocellular carcinoma; STAD, stomach adenocarcinoma; ACC, adrenocortical carcinoma; BLCA, bladder urothelial carcinoma; CESC, cervical squamous cell carcinoma and endocervical adenocarcinoma; CHOL, cholangiocarcinoma; COAD, colon adenocarcinoma; DLBC, lymphoid neoplasm diffuse large B-cell lymphoma; KICH, kidney chromophobe; KIRC, kidney renal clear cell carcinoma; KIRP, kidney renal papillary cell carcinoma; LAML, acute myeloid leukemia; LUAD, lung adenocarcinoma; LUSC, lung squamous cell carcinoma; MESO, mesothelioma; OV, ovarian serous cystadenocarcinoma; PAAD, pancreatic adenocarcinoma; PCPG, pheochromocytoma and paraganglioma; PRAD, prostate adenocarcinoma; READ, rectum adenocarcinoma; SARC, sarcoma; SKCM, skin cutaneous melanoma; TGCT, testicular germ cell tumours; THCA, thyroid carcinoma; THYM, thymoma; UCS, uterine carcinosarcoma; UVM, uveal melanoma. 
A

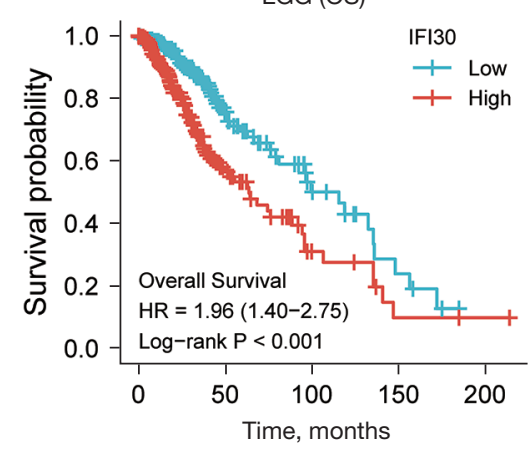

B

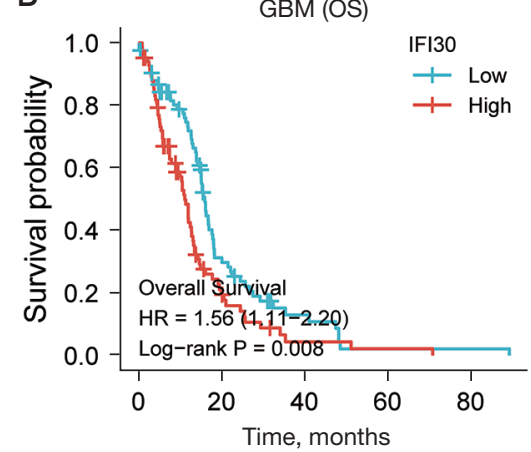

C

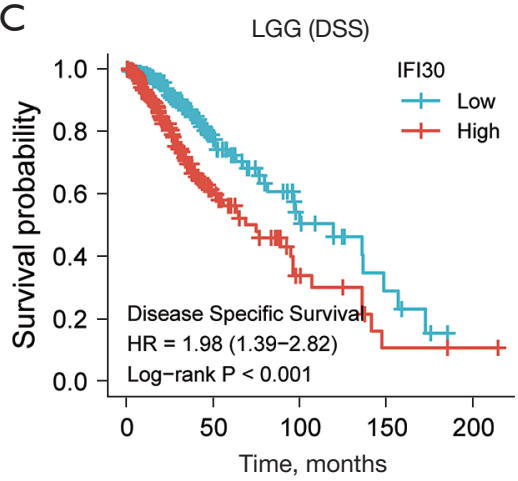

D

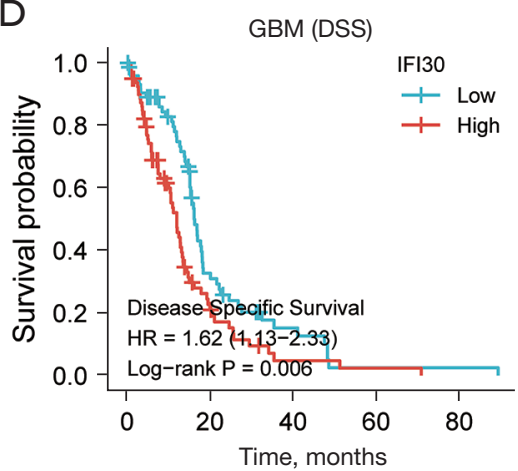

E

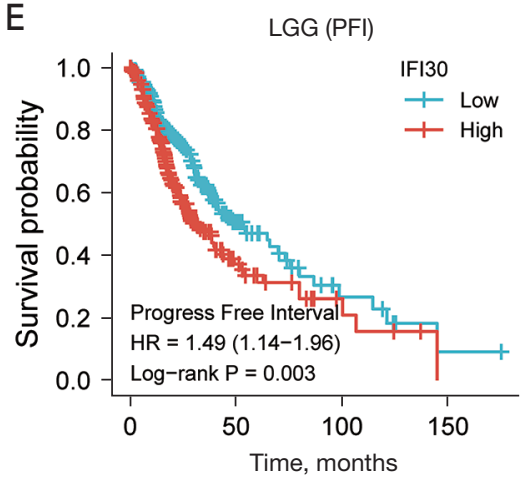

$\mathrm{F}$

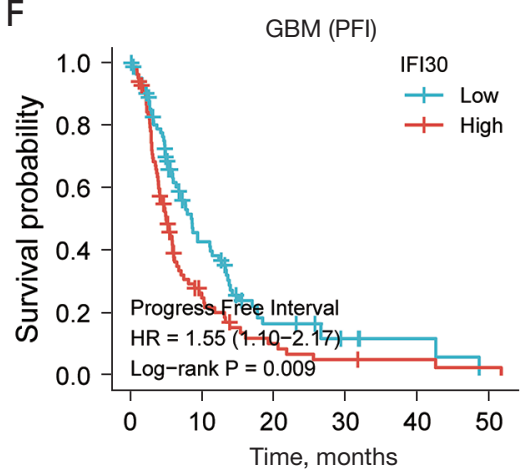

Figure 2 Survival analysis of high and low IFI30 gene expression in glioma (LGG and GBM). (A,B) The relationship between IFI30 gene expression in LGG and GBM and OS. (C,D) The relationship between IFI30 gene expression in LGG and GBM and DSS. (E,F) The relationship between IFI30 gene expression in LGG and GBM and PFI. IFI30, interferon-gamma-inducible protein 30; LGG, low grade glioma; GBM, glioblastoma multiforme; OS, overall survival; DSS, disease-specific survival; PFI, progression-free interval.

group was 5 (4.1-6.2) months and $8.6(6.8-12.3)$ months for the low IFI30 group in GBM (Figure $2 F$ ).

Univariate Cox regression analysis showed that age, WHO grade and IFI30 were significantly associated with the prognosis of LGG. Multivariate Cox regression analysis confirmed that IFI30 was an independent prognostic factor after adjusting for other clinicopathologic factors (Table 1). Univariate and multivariate Cox regression analyses showed IFI30 was also an independent prognostic factor and significantly associated with the prognosis of GBM (Table 2).

\section{Relationship between IFI30 expression and clinical characteristics in LGG}

Clinical and gene expression data of LGG patients were downloaded from the TCGA database. Patients were divided into high and low expression groups on the basis of median IFI30 mRNA expression. The relationship between clinical characteristics and IFI30 mRNA expression in LGG was analysed and the results revealed that IFI30 mRNA expression was statistically associated with WHO grade, isocitrate dehydrogenase (IDH) status and $1 \mathrm{p} / 19 \mathrm{q}$ codeletion (all $\mathrm{P}<0.001$; Table 3).

As shown in Figure $3 A, 3 B$, there was no difference in the expression level of IFI30 in LGG patients of different sex or age. The expression level of IFI30 in IDH wildtype LGG patients was higher than that in IDH wildtype (Figure 3C). The expression level of IFI30 in G3 glioma patients was significantly higher than that in G2 patients, and the expression level of IFI30 in G4 glioma patients was significantly higher than that in G2 and G3 patients (Figure 3D,3E). The expression level of IFI30 in $1 \mathrm{p} / 19 \mathrm{q}$ non-codel LGG patients was higher than that of 1p/19q codel (Figure 3F). IFI30 expression was higher in LGG patients who progressed after initial treatment than in LGG patients who were stable after initial treatment (Figure 3G). 
Table 1 Univariate and multivariate Cox analyses of clinicopathologic factors and IFI30 in LGG

\begin{tabular}{|c|c|c|c|c|c|}
\hline Characteristic & Total (n) & \multicolumn{2}{|c|}{ Univariate analysis } & \multicolumn{2}{|c|}{ Multivariate analysis } \\
\hline Sex (female vs. male) & 527 & $0.889(0.633-1.250)$ & 0.499 & & \\
\hline $\begin{array}{l}\text { Race (Asian \& Black or } \\
\text { African American vs. White) }\end{array}$ & 516 & $1.178(0.549-2.529)$ & 0.675 & & \\
\hline Age (>40 vs. $\leq 40)$ years & 527 & 2.889 (2.009-4.155) & $<0.001$ & 3.149 (2.068-4.794) & $<0.001$ \\
\hline
\end{tabular}

Univariate variables with $\mathrm{P}<0.05$ were chosen for the multivariate analysis. IFI30, interferon-gamma-inducible protein 30; LGG, low grade glioma; $\mathrm{Cl}$, confidence interval.

Table 2 Univariate and multivariate Cox analyses of clinicopathologic factors and IFI30 in GBM

\begin{tabular}{|c|c|c|c|c|c|}
\hline Characteristic & Total (n) & \multicolumn{2}{|c|}{ Univariate analysis } & \multicolumn{2}{|c|}{ Multivariate analysis } \\
\hline Sex (male vs. female) & 168 & $1.026(0.719-1.466)$ & 0.887 & & \\
\hline $\begin{array}{l}\text { Race (White vs. Asian \& } \\
\text { Black or African American) }\end{array}$ & 166 & $0.864(0.451-1.653)$ & 0.658 & & \\
\hline IFI30 (high vs. low) & 168 & $1.581(1.124-2.224)$ & 0.009 & $1.581(1.124-2.224)$ & 0.009 \\
\hline
\end{tabular}

IFI30, interferon-gamma-inducible protein 30; GBM, glioblastoma multiforme; Cl, confidence interval.

\section{Relationship between IFI30 expression and ImmuneScore of various tumours}

Because of reports indicating that the tumour immune microenvironment plays an essential role in the occurrence and development of tumours, we used the $\mathrm{R}$ software package to estimate the ImmuneScore of various tumour samples and observe the IFI30 gene expression and ImmuneScore in 33 tumours. As shown in Figure 4, IFI30 was positively associated with an immune score in all tumours except thymoma (THYM).

\section{Expression correlation of IFI30 and biomarkers of immune cells in LGG}

As shown in Figure $5 \mathrm{~A}$, the arm-level gain copy number of IFI30 was associated with the level of immune cell infiltration (including $\mathrm{B}$ cells, $\mathrm{CD} 4^{+} \mathrm{T}$ cells, macrophages, neutrophils and dendritic cells) in LGG. The expression level of IFI30 significantly positively correlated with the level of infiltration of $\mathrm{B}$ cells, $\mathrm{CD}^{+} \mathrm{T}$ cells, $\mathrm{CD} 8^{+} \mathrm{T}$ cells, macrophages, neutrophils and dendritic cells in LGG (Figure 5B). Cumulative survival analysis showed high expression of IFI30, B cells, CD4 ${ }^{+} \mathrm{T}$ cells, $\mathrm{CD} 8^{+} \mathrm{T}$ cells, macrophages, neutrophils and dendritic cells was associated with poor prognosis in LGG (Figure 5C).

\section{Relationship between IFI30 expression and immune checkpoint in glioma}

Immune checkpoint molecules expressed on immune cells will inhibit the function of immune cells such that the body cannot produce an effective anti-tumour immune response and there is immune escape of tumour formation. SIGLEC15, TIGIT, CD274, HAVCR2, PDCD1, CTLA4, $L A G 3$ and $P D C D 1 L G 2$ are immune checkpoint-related transcripts. We extracted the expression values of these eight genes and observed the differences in their expression in glioma tissues with high and low IFI30 expression levels. Expression of IFI30 correlated positively with all eight immune checkpoints, showing statistical significance in LGG (Figure 6A) and GBM (Figure 6B). 
Table 3 Relationship between IFI30 expression and clinical characteristics in LGG ( $\mathrm{n}=528)$

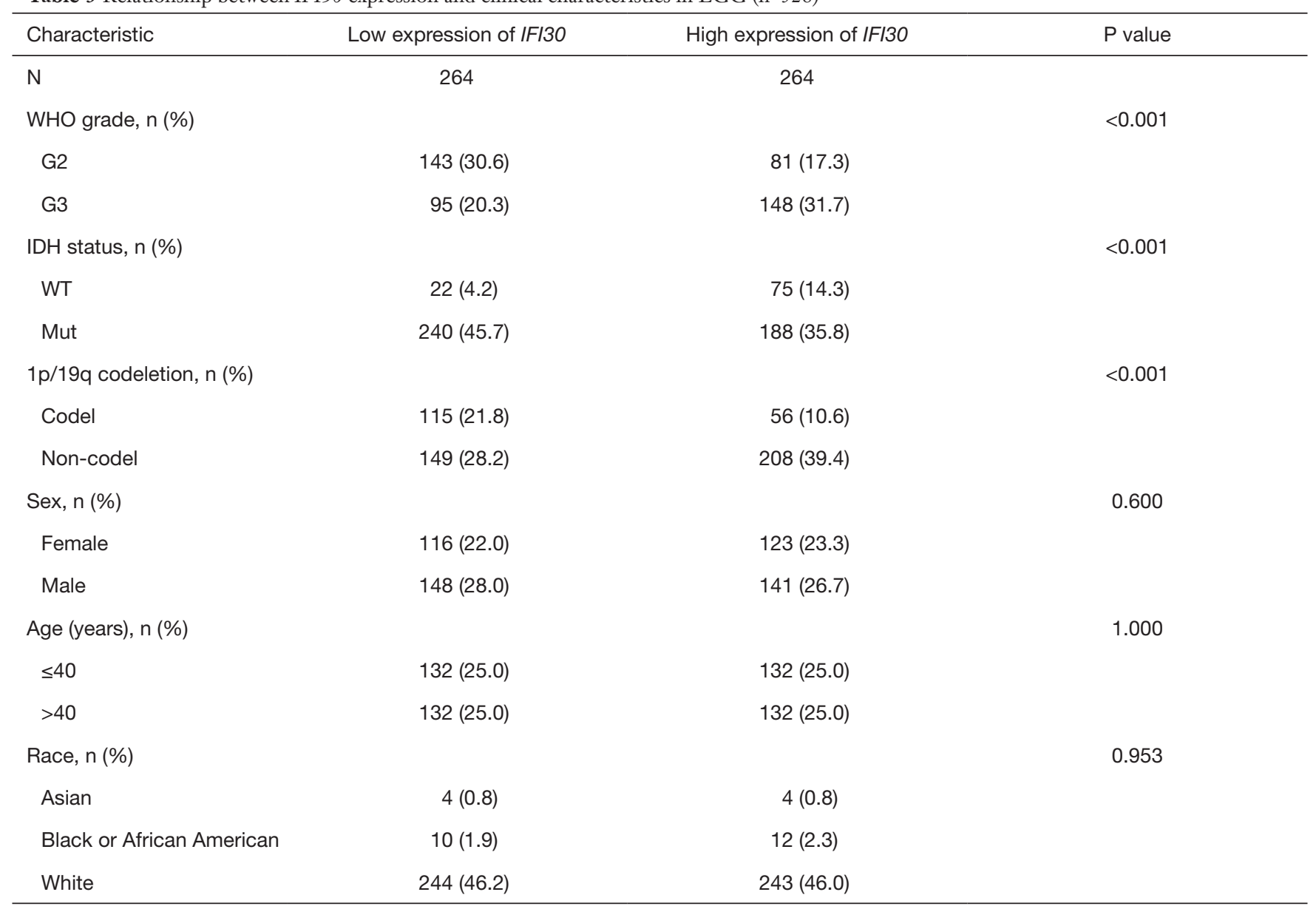

IFI30, interferon-gamma-inducible protein 30; LGG, low grade glioma; IDH, isocitrate dehydrogenase.

\section{Functional enrichment and analyses of IFI30 similar genes in glioma}

We used the GEPIA online tool to analyse 120 genes similar to IFI30 in gliomas (LGG and GBM). Next, these significantly similar genes were chosen for GO and KEGG analysis using DAVID (https://david.ncifcrf.gov/). As shown in Table 4, IFI30 similar genes are involved in many biological processes (BPs), cellular compositions (CCs), molecular functions (MFs), and KEGG pathways, including neutrophil degranulation, neutrophil activation involved in immune responses, neutrophil activation, neutrophilmediated immunity, regulation of innate immune response, secretory granule membrane, tertiary granules, secretory granule lumen, tertiary granule membrane, cytoplasmic vesicle lumen, IgG binding, cysteine-type endopeptidase activity, immunoglobulin binding, RAGE receptor binding, Toll-like receptor binding, Staphylococcus aureus infection, osteoclast differentiation, phagosome, pertussis and leishmaniasis.

\section{Gene sets enriched in IFI30 expression phenotype}

In order to evaluate the function of IFI30-associated DEGs in glioma patients, GSEA analysis was applied based on TCGA-LGG cohort. Strikingly, we identified enrichment for several hallmark inflammation signatures, such as inflammatory response and interferon-alpha response (Figure $7 A, 7 B$ ). The GSEA showed IFI30-associated DEGs significantly enriched in hallmark tumour progressionrelated clusters, such as angiogenesis $(\mathrm{NES}=1.984$; adjusted $\mathrm{P}=0.003$; FDR $=0.002$; Figure $7 C$ ), and epithelial mesenchymal transition $(\mathrm{NES}=2.195$; adjusted $\mathrm{P}=0.003$; $\mathrm{FDR}=0.002$; Figure $7 D$ ). The IFI30-associated DEGs were also enriched in cancer pathways, such as IL6/JAK/STAT3 

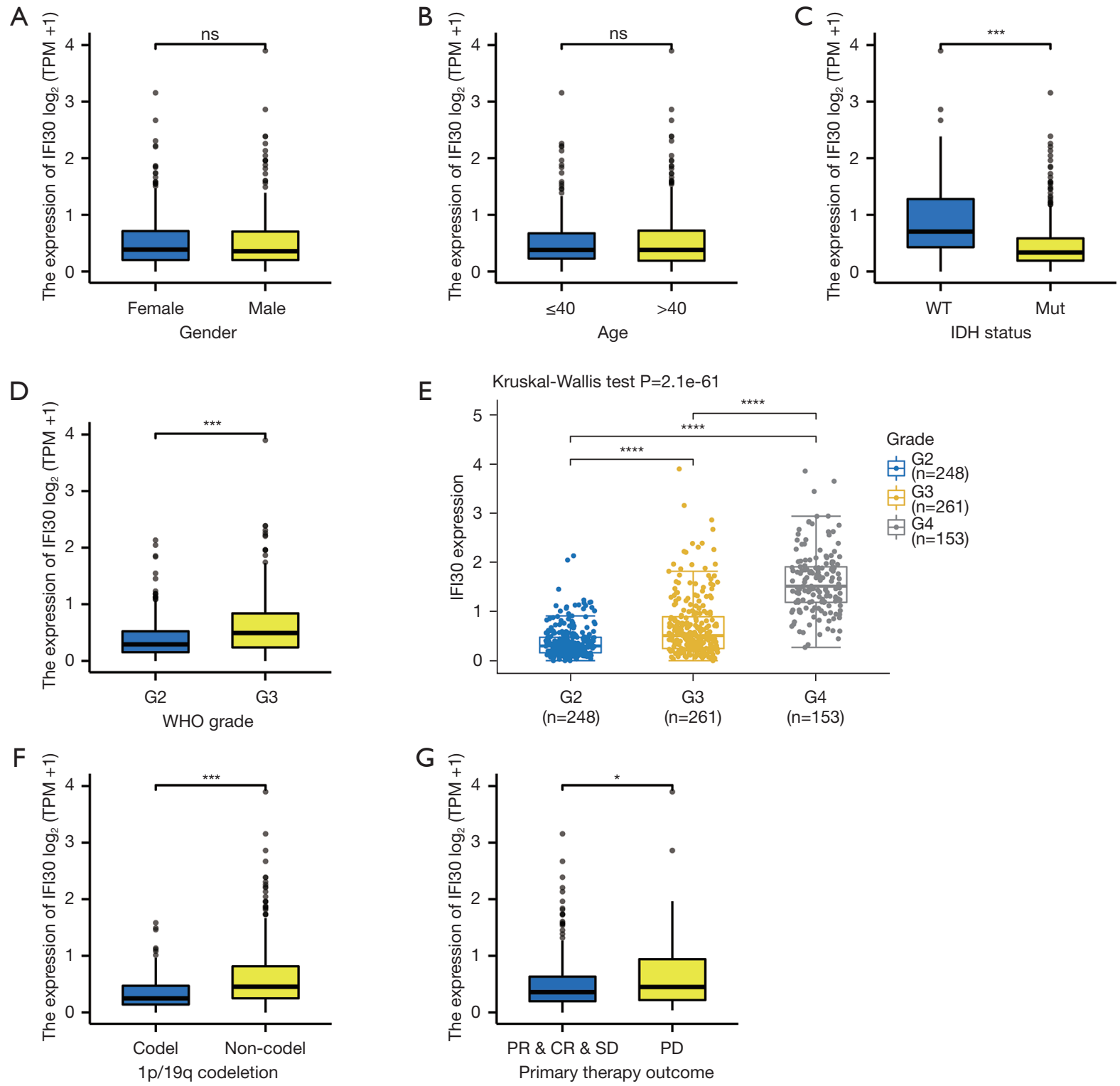

Figure 3 Distribution of the IFI30 in different clinicopathologic features of glioma. (A-F) Sex, age, IDH status, grade, 1p/19q codeletion and IFI30 expression level of LGG patients with initial treatment evaluation subgroup. (G) IFI30 expression levels in gliomas of different grades (LGG and GBM). *, P value $\leq 0.05 ;{ }^{* * *}$, P value $\leq 0.001 ;{ }^{* * *}$, $\mathrm{P}$ value $\leq 0.0001 ;$ ns, $\mathrm{P}$ value $>0.05$. IFI30, interferon-gamma-inducible protein 30; IDH, isocitrate dehydrogenase; LGG, low grade glioma; GBM, glioblastoma multiforme; TPM, transcripts per million.

signalling $(\mathrm{NES}=2.205$; adjusted $\mathrm{P}=0.003 ; \mathrm{FDR}=0.002$; Figure $7 E$ ), and TNFA signalling via NF- $\mathrm{kB}(\mathrm{NES}=2.263$; adjusted $\mathrm{P}=0.003 ;$ FDR $=0.002 ;$ Figure $7 F$ ).

\section{Discussion}

Glioma is the most common primary tumour of the central nervous system and has a poor prognosis.
Tumour-infiltrating immune cells (TIIC) exist in the microenvironment of glioma, and their composition and proportion correlate with the prognosis of patients. Increasing evidence has demonstrated that IFI30 is a prognostic marker and plays key roles in the progression of multiple human cancers, including glioma. However, understanding of the role of IFI3O in the immune microenvironment of glioma remains inadequate and needs 


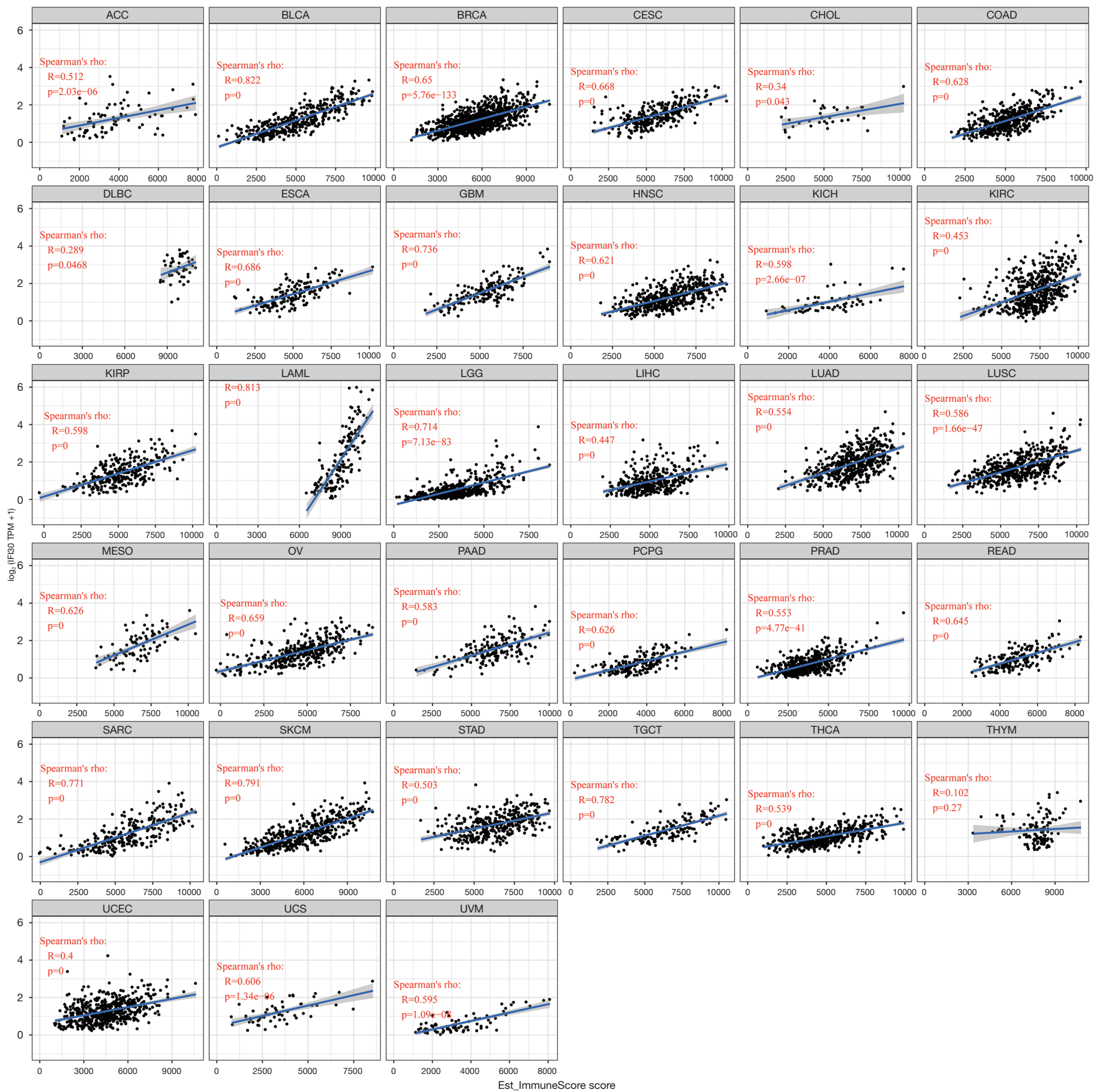

Figure 4 Relationship between IFI30 expression and ImmuneScore of the 33 various tumour. IFI30, interferon-gamma-inducible protein 30; ACC, adrenocortical carcinoma; BLCA, bladder urothelial carcinoma; BRCA, breast invasive carcinoma; CESC, cervical squamous cell carcinoma and endocervical adenocarcinoma; CHOL, cholangiocarcinoma; COAD, colon adenocarcinoma; DLBC, lymphoid neoplasm diffuse large B-cell lymphoma; ESCA, esophageal carcinoma; GBM, glioblastoma multiforme; HNSC, head and neck squamous cell carcinoma; KICH, kidney chromophobe; KIRC, kidney renal clear cell carcinoma; KIRP, kidney renal papillary cell carcinoma; LAML, acute myeloid leukemia; LGG, lower grade glioma; LIHC, liver hepatocellular carcinoma; LUAD, lung adenocarcinoma; LUSC, lung squamous cell carcinoma; MESO, mesothelioma; OV, ovarian serous cystadenocarcinoma; PAAD, pancreatic adenocarcinoma; PCPG, pheochromocytoma and paraganglioma; PRAD, prostate adenocarcinoma; READ, rectum adenocarcinoma; SARC, sarcoma; SKCM, skin cutaneous melanoma; STAD, stomach adenocarcinoma; TGCT, testicular germ cell tumours; THCA, thyroid carcinoma; THYM, thymoma; UCEC, uterine corpus endometrial carcinoma; UCS, uterine carcinosarcoma; UVM, uveal melanoma. 
A

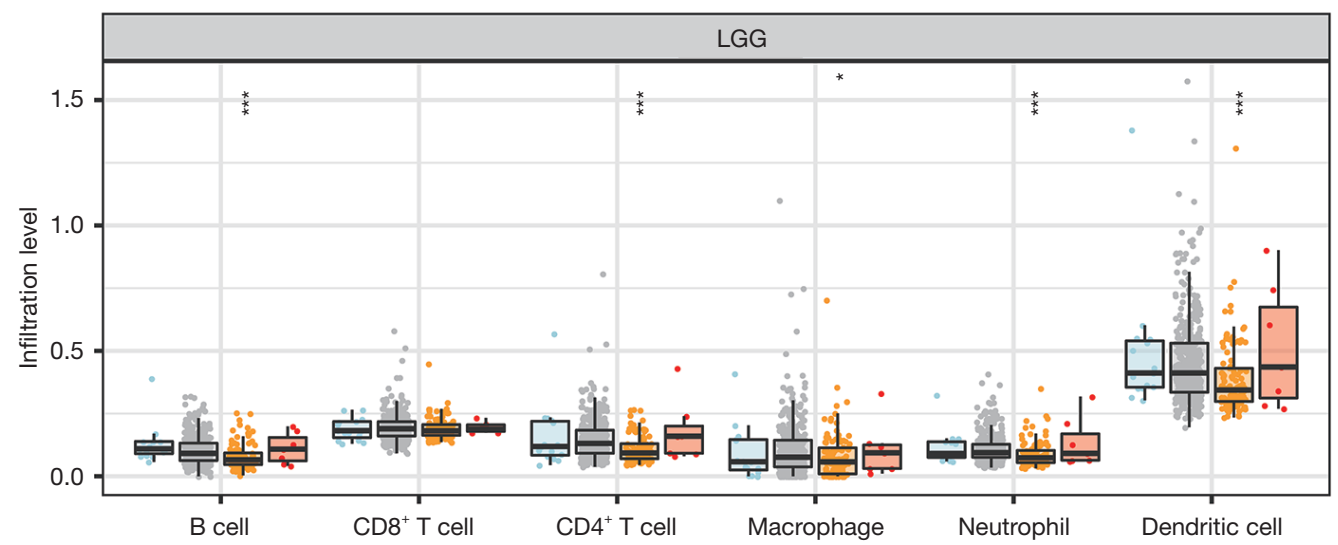

Copy number

由 Arm-level deletion

审 Diploid/normal

审 Arm-level gain

审 High amplication

B
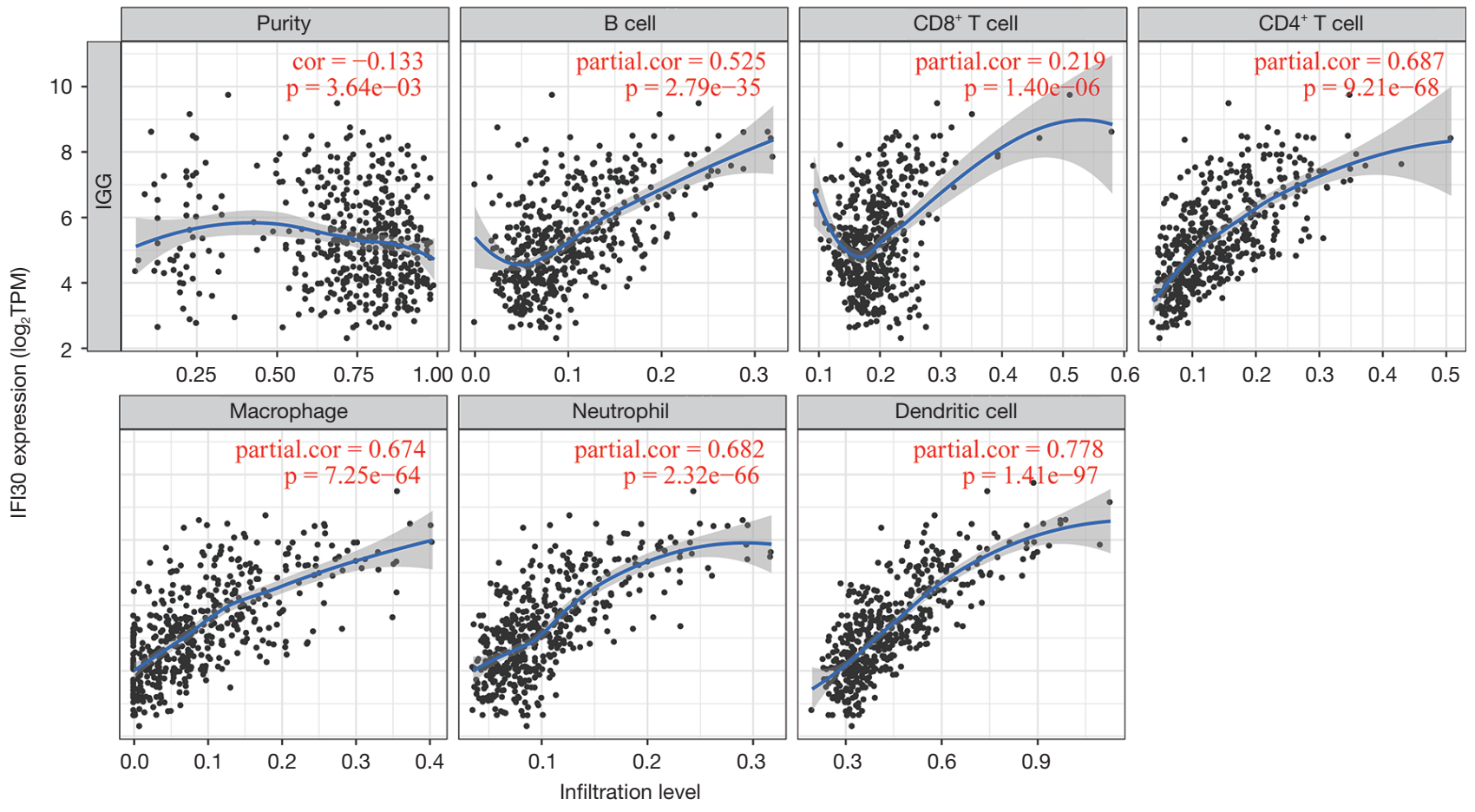

C
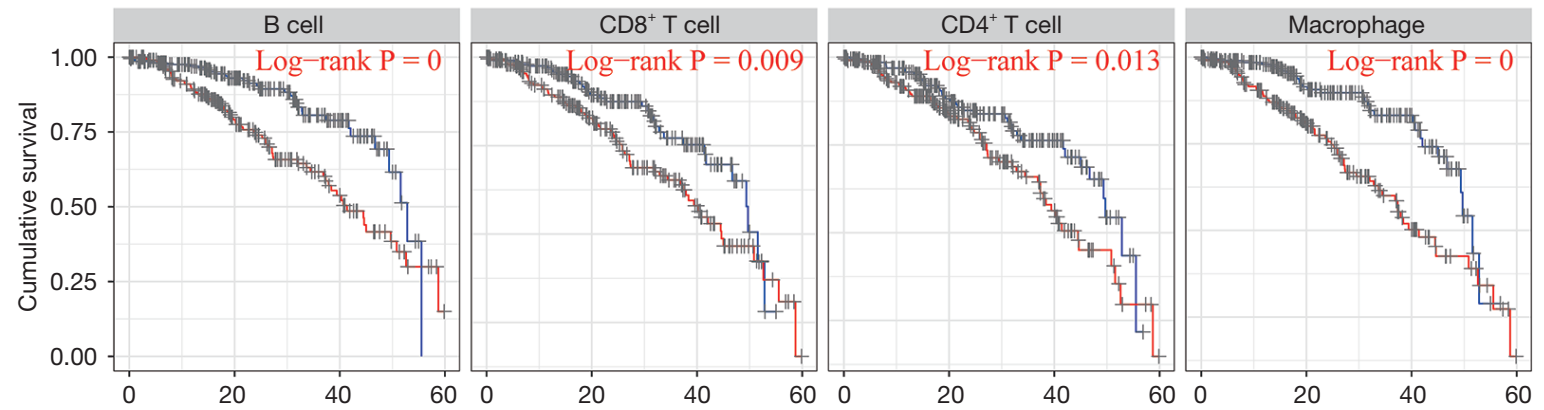

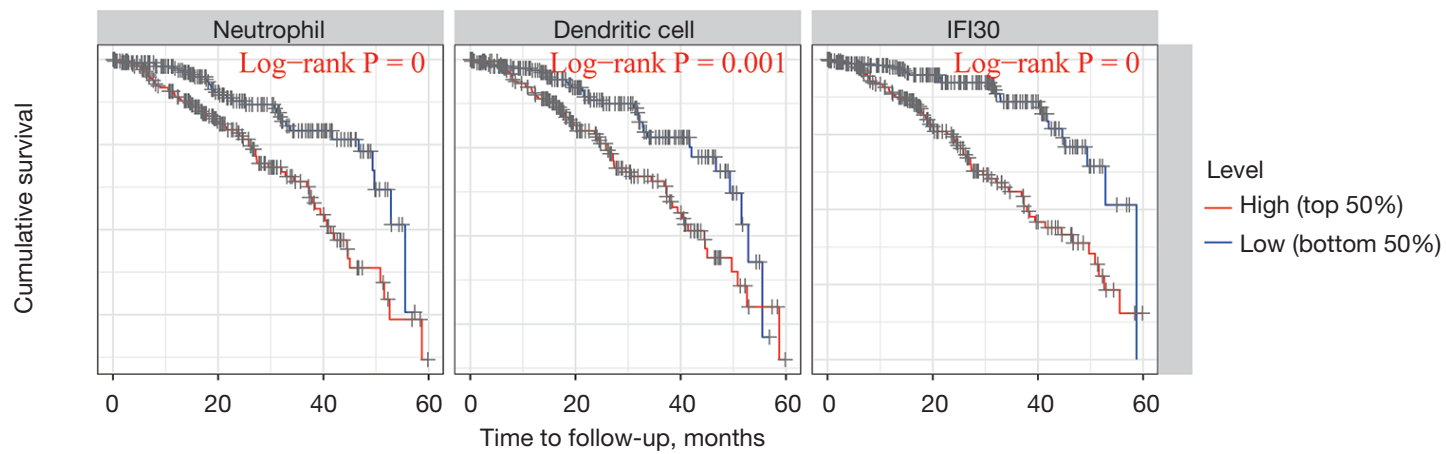

Figure 5 Relationship between immune cell infiltration and IFI30 level in LGG. (A) Level of infiltration of various immune cells under different copy numbers of IFI30 in LGG. (B) Correlation of IFI30 expression level with level of infiltration of B cells, CD8 ${ }^{+}$T cells, CD4 ${ }^{+}$T cells, macrophages, neutrophils, or dendritic cells in LGG. (C) Cumulative survival analysis of IFI30 expression and level of infiltration of B cells, $\mathrm{CD}^{+} \mathrm{T}$ cells, CD4 ${ }^{+} \mathrm{T}$ cells, macrophages, neutrophils, and dendritic cells in LGG. * $\mathrm{P}<0.05$; ${ }^{* * *}, \mathrm{P}<0.001$. IFI30, interferon-gammainducible protein 30; LGG, low grade glioma; TPM, transcripts per million.

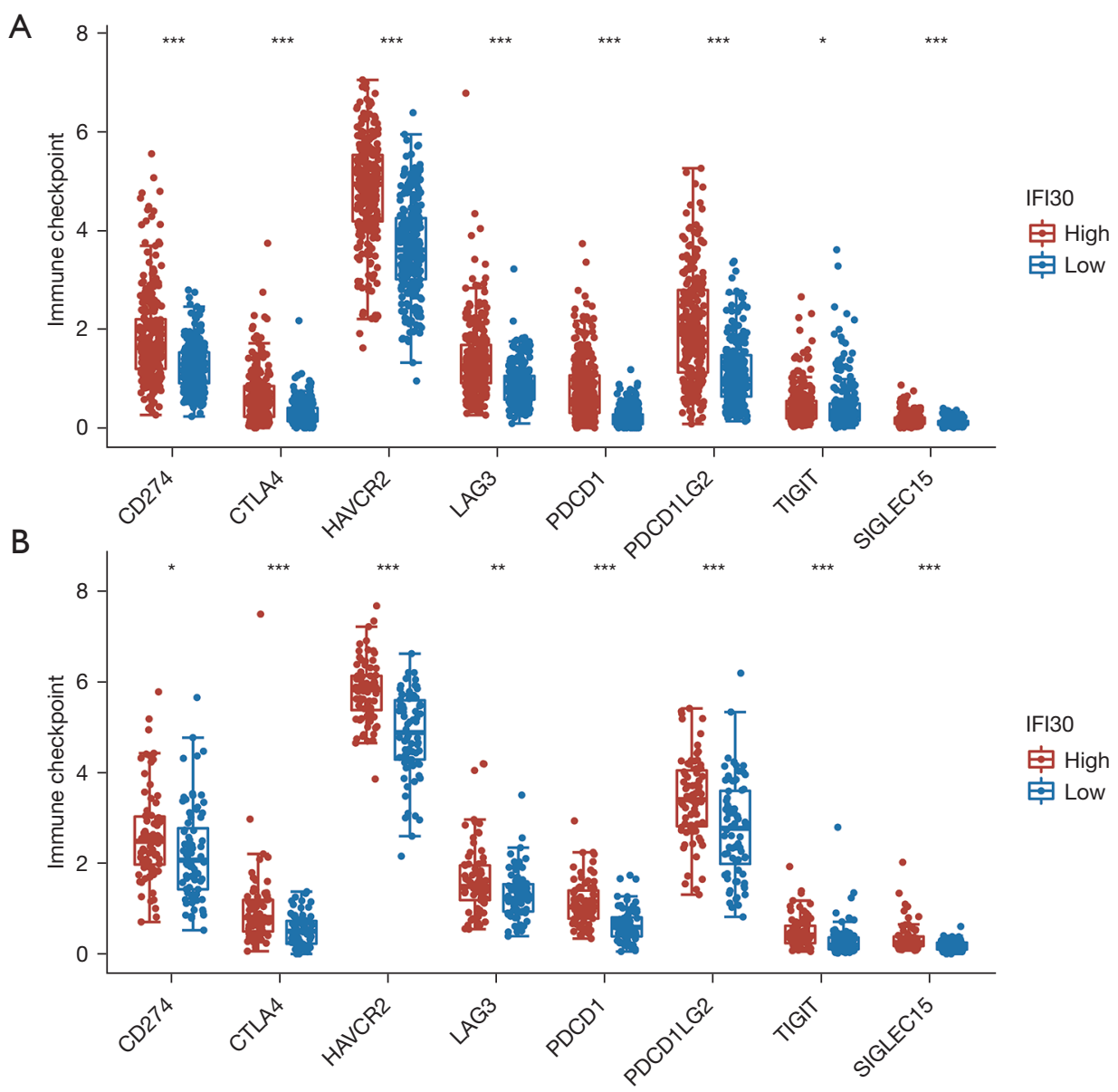

Figure 6 Relationship of immune checkpoint with IFI30 expression in LGG. Expression of IFI30 in (A) LGG tissues and (B) GBM tissues. The horizontal axis represents different groups of samples, and the vertical axis represents the gene expression distribution, where different colours represent different groups. The upper left corner represents the significance $\mathrm{P}$ value test method. ${ }^{*}, \mathrm{P}<0.05 ;{ }^{* *}, \mathrm{P}<0.01 ;{ }^{* * *}, \mathrm{P}<0.001$. IFI30, interferon-gamma-inducible protein 30; LGG, low grade glioma; GBM, glioblastoma multiforme. 
Table 4 Significantly enriched GO annotations and KEGG pathways of IFI30 similar genes in glioma

\begin{tabular}{|c|c|c|c|c|c|}
\hline Ontology & Description & GeneRatio & BgRatio & $P$ adjust & $P$ value \\
\hline $\mathrm{BP}$ & Neutrophil activation involved in immune response & $37 / 109$ & $488 / 18,670$ & $2.06 e-28$ & $1.51 \mathrm{e}-28$ \\
\hline BP & Neutrophil activation & $37 / 109$ & $498 / 18,670$ & $2.33 e-28$ & $1.70 \mathrm{e}-28$ \\
\hline BP & Neutrophil-mediated immunity & $37 / 109$ & $499 / 18,670$ & $2.33 e-28$ & $1.70 \mathrm{e}-28$ \\
\hline $\mathrm{CC}$ & Secretory granule membrane & $19 / 113$ & $298 / 19,717$ & $1.06 e-12$ & $7.35 \mathrm{e}-13$ \\
\hline $\mathrm{CC}$ & Tertiary granule & $15 / 113$ & $164 / 19,717$ & $2.30 e-12$ & $1.60 \mathrm{e}-12$ \\
\hline CC & Secretory granule lumen & $18 / 113$ & $321 / 19,717$ & $1.37 e-11$ & $9.55 \mathrm{e}-12$ \\
\hline $\mathrm{CC}$ & Tertiary granule membrane & $11 / 113$ & $73 / 19,717$ & $1.37 e-11$ & $9.55 \mathrm{e}-12$ \\
\hline MF & Cysteine-type endopeptidase activity & $7 / 105$ & $116 / 17,697$ & $8.10 e-04$ & $6.94 \mathrm{e}-04$ \\
\hline MF & Immunoglobulin binding & $4 / 105$ & $24 / 17,697$ & 0.001 & $9.04 \mathrm{e}-04$ \\
\hline MF & RAGE receptor binding & $3 / 105$ & $11 / 17,697$ & 0.002 & 0.002 \\
\hline MF & Toll-like receptor binding & $3 / 105$ & $12 / 17,697$ & 0.002 & 0.002 \\
\hline KEGG & Staphylococcus aureus infection & $12 / 72$ & $96 / 8,076$ & $4.58 e-09$ & $3.55 \mathrm{e}-09$ \\
\hline KEGG & Osteoclast differentiation & $13 / 72$ & $128 / 8,076$ & $4.70 \mathrm{e}-09$ & $3.64 \mathrm{e}-09$ \\
\hline KEGG & Phagosome & $12 / 72$ & $152 / 8,076$ & $3.37 e-07$ & $2.61 e-07$ \\
\hline KEGG & Pertussis & $9 / 72$ & $76 / 8,076$ & $6.41 \mathrm{e}-07$ & $4.96 \mathrm{e}-07$ \\
\hline
\end{tabular}

GO, Gene Ontology; KEGG, Kyoto Encyclopedia of Genes and Genomes; IF/30, interferon-gamma-inducible protein 30; BP, biological process; CC, cellular composition; MF, molecular function.

further investigation.

In this study, we used TCGA pan-cancer data to analyse the expression of IFI30 in both tumour and normal tissues. Survival analysis for IFI30 indicated that glioma patients with high expression of IFI30 had poor OS, DSS and PFI. Although previous studies have reported that overexpression of IFI30 is an adverse prognostic factor for any tumour, including glioma, previous studies only analysed OS. Considering the possibility of non-tumour death during follow-up, we also analysed the relationship between IFI30 gene expression and DSS in glioma. Therefore, we identified IFI30 as a marker of poor prognosis of glioma in a more systematic and comprehensive way. In vitro experiments showed that Gilt silencing suppresses cell proliferation, colony formation, migration, and tumour growth and induces apoptosis and cell cycle arrest in glioma.
That report, together with our analytic results, show the oncogenic role of IFI30 in glioma.

Immune infiltrates in the TME play a crucial role in tumour development and could influence the efficacy of chemotherapy, radiotherapy, and immunotherapy and thus the prognosis of tumour patients. A comprehensive analysis of TIIC will shed light on the mechanisms of tumour immune escape and thus provide an opportunity to develop new therapeutic strategies (20). Our work showed IFI30 was significantly positively correlated with B cells, $\mathrm{CD}^{+} \mathrm{T}$ cells, $\mathrm{CD}^{+} \mathrm{T}$ cells, macrophages, neutrophils, and dendritic cells in LGG. Cumulative survival analysis showed high expression of $\mathrm{B}$ cells, $\mathrm{CD} 4^{+} \mathrm{T}$ cells, $\mathrm{CD} 8^{+}$ $\mathrm{T}$ cells, macrophages, neutrophils and dendritic cells was associated with poor prognosis in LGG. Moreover, IFI30 was markedly positively associated with the immune score 
A

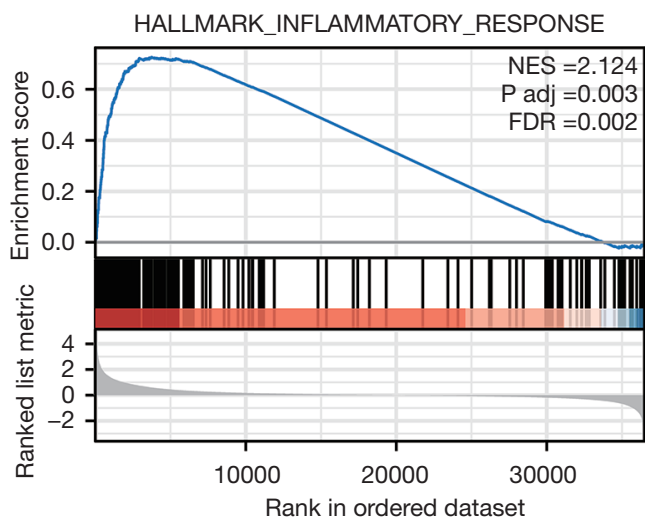

C

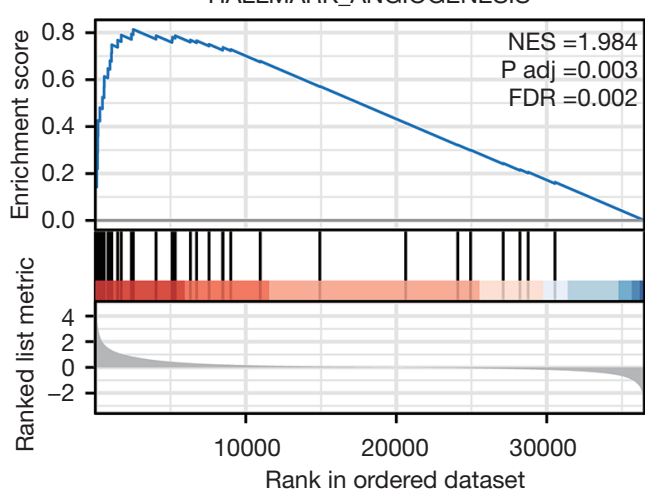

$\mathrm{E}$

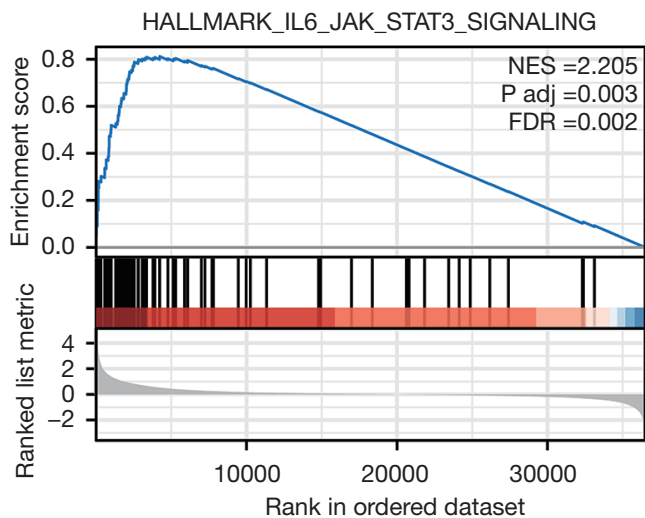

B

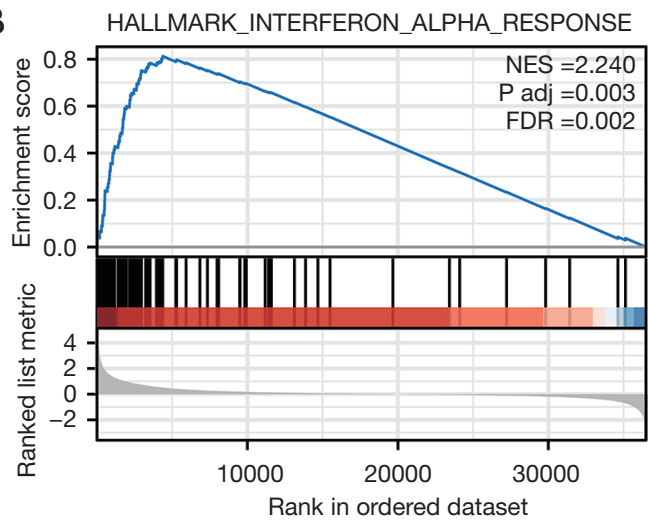

D HALLMARK_EPITHELIAL_MESENCHYMAL_TRANSITION

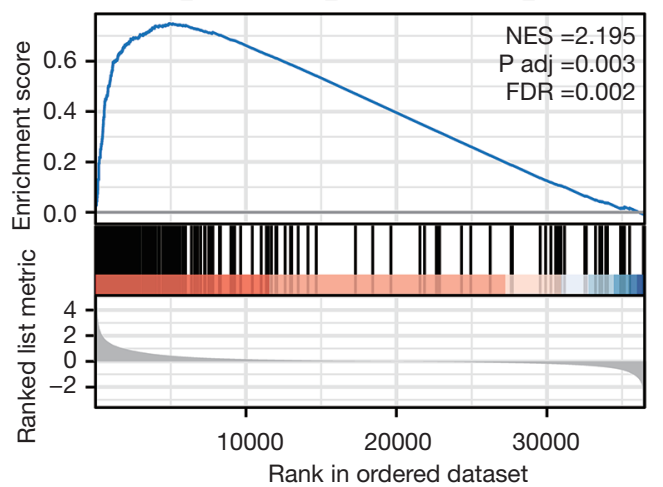

$\mathrm{F}$

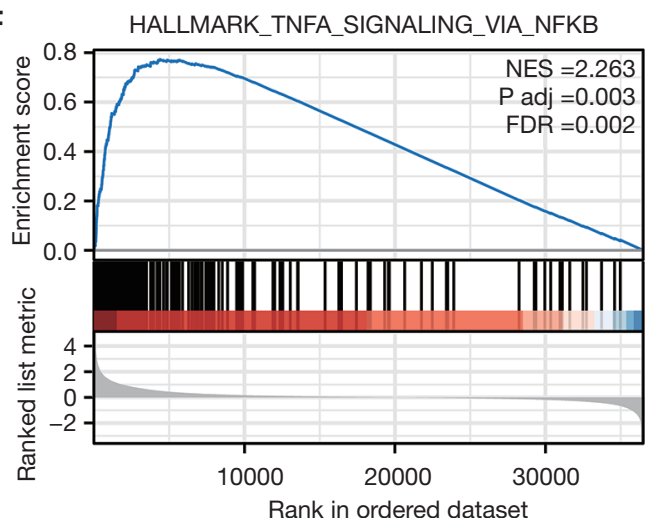

Figure 7 Enrichment plots of GSEA. (A-F) Functional annotation of DEGs in LGG patients with high- and low-IFI30 expression. GSEA, gene set enrichment analysis; DEGs, differentially expressed genes; LGG, low grade glioma; IFI30, interferon-gamma-inducible protein 30; NES, normalised enrichment score; FDR, false discovery rate.

in almost all 33 tumours sampled. These findings indicated that tumour immune infiltration partially accounted for the IFI30-mediated oncogenic role in glioma.

Immune checkpoints are negative regulators that inhibit the proliferation and activity of $\mathrm{T}$ cells $(21,22)$, and are often used by cancer cells to avoid immune surveillance $(23,24)$. Thus, we also assessed the relationship between
IFI30 and immune checkpoints and found that high expression of IFI30 was linked to SIGLEC15, TIGIT, CD274, HAVCR2, PDCD1, CTLA4, LAG3 and PDCD1LG2 in glioma. To date, this is the first report of an association between IFI30 and immune checkpoints. Our findings indicated that avoidance of immune surveillance partially accounted for IFI30-mediated carcinogenesis in glioma and 
targeting IFI30 took happens the efficacy of immunotherapy in glioma.

Functional enrichment analyses showed that IFI30 similar genes were involved in neutrophil activation, neutrophil-mediated immunity, IgG binding, cysteinetype endopeptidase activity, immunoglobulin binding, tolllike receptor binding. In addition, GSEA analyses showed that IFI30-associated genes were significantly enriched in tumour progression-related pathways, such as angiogenesis, epithelial mesenchymal transition, IL6/JAK/STAT3 signalling, and tumour necrosis factor-alpha signalling via NF-кB. The IL-6/JAK/STAT3 pathway is involved in the pathogenesis of many human malignancies (25). STAT3 has also been shown to regulate the self-renewal potential of glioma cells (26). NF- $\mathrm{KB}$ signalling regulates the expression of several molecules participating in various crucial physiological reactions, and has been demonstrated to act as a critical factor in the development or progression of various cancers, as well as glioma (27-29). NF- $\mathrm{BB}$ activation is also prevalent in carcinomas, in which NF- $\mathrm{KB}$ activation is mainly driven by inflammatory cytokines within the TME (30). These findings indicated that IFI30 acted as a regulator of key signalling pathways in glioma.

Returning to the question posed at the beginning of this study, it is now possible to state that IFI30 was highly expressed in multiple types of human cancer and positively correlated with unfavourable prognosis in glioma. Furthermore, IFI30 might exert its oncogenic role through increasing tumour immune cell infiltration, immune checkpoint expression and regulation of tumour progression-related pathways. However, these results need to be validated by more basic experiments in the future.

\section{Acknowledgments}

Funding: This study was supported by grants from the National Natural Science Foundation (82070622, 81702419), the Key Research and Development Plan of Jiangsu Province (BE2020668), and the Nantong Science and Technology Project (MS12019013).

\section{Footnote}

Reporting Checklist: The authors have completed the REMARK reporting checklist. Available at https://dx.doi. org/10.21037/atm-21-5569

Conflicts of Interest: All authors have completed the ICMJE uniform disclosure form (available at https://dx.doi. org/10.21037/atm-21-5569). The authors have no conflicts of interest to declare.

Ethical Statement: The authors are accountable for all aspects of the work in ensuring that questions related to the accuracy or integrity of any part of the work are appropriately investigated and resolved. The study was conducted in accordance with the Declaration of Helsinki (as revised in 2013).

Open Access Statement: This is an Open Access article distributed in accordance with the Creative Commons Attribution-NonCommercial-NoDerivs 4.0 International License (CC BY-NC-ND 4.0), which permits the noncommercial replication and distribution of the article with the strict proviso that no changes or edits are made and the original work is properly cited (including links to both the formal publication through the relevant DOI and the license). See: https://creativecommons.org/licenses/by-nc-nd/4.0/.

\section{References}

1. Zhang B, Chang K, Ramkissoon S, et al. Multimodal MRI features predict isocitrate dehydrogenase genotype in high-grade gliomas. Neuro Oncol 2017;19:109-17.

2. Melin BS, Barnholtz-Sloan JS, Wrensch MR, et al. Genome-wide association study of glioma subtypes identifies specific differences in genetic susceptibility to glioblastoma and non-glioblastoma tumors. Nat Genet 2017;49:789-94.

3. Ostrom QT, Gittleman H, Fulop J, et al. CBTRUS statistical report: primary brain and central nervous system tumors diagnosed in the United States in 2008-2012. Neuro Oncol 2015;17 Suppl 4:iv1-62.

4. Weller M, Wick W, Aldape K, et al. Glioma. Nat Rev Dis Primers 2015;1:15017.

5. Wick W, Dettmer S, Berberich A, et al. N2M2 (NOA-20) phase I/II trial of molecularly matched targeted therapies plus radiotherapy in patients with newly diagnosed nonMGMT hypermethylated glioblastoma. Neuro Oncol 2019;21:95-105.

6. Aran D, Sirota M, Butte AJ. Systematic pan-cancer analysis of tumour purity. Nat Commun 2015;6:8971.

7. Zhao X, Ding L, Lu Z, et al. Diminished CD68+ cancerassociated fibroblast subset induces regulatory T-cell (Treg) infiltration and predicts poor prognosis of oral squamous cell carcinoma patients. Am J Pathol 2020;190:886-99. 
8. Cooks T, Pateras IS, Jenkins LM, et al. Mutant p53 cancers reprogram macrophages to tumor supporting macrophages via exosomal miR-1246. Nat Commun 2018;9:771.

9. Colegio OR, Chu NQ, Szabo AL, et al. Functional polarization of tumour-associated macrophages by tumourderived lactic acid. Nature 2014;513:559-63.

10. Wang JJ, Lei KF, Han F. Tumor microenvironment: recent advances in various cancer treatments. Eur Rev Med Pharmacol Sci 2018;22:3855-64.

11. Louveau A, Herz J, Alme MN, et al. CNS lymphatic drainage and neuroinflammation are regulated by meningeal lymphatic vasculature. Nat Neurosci 2018;21:1380-91.

12. Buetow KH, Meador LR, Menon H, et al. High GILT expression and an active and intact MHC class II antigen presentation pathway are associated with improved survival in melanoma. J Immunol 2019;203:2577-87.

13. Rausch MP, Hastings KT. GILT modulates CD4+ T-cell tolerance to the melanocyte differentiation antigen tyrosinase-related protein 1 . J Invest Dermatol 2012;132:154-62.

14. West LC, Cresswell P. Expanding roles for GILT in immunity. Curr Opin Immunol 2013;25:103-8.

15. Zhou C, Wei Z, Zhang L, et al. Systematically Characterizing A-to-I RNA Editing Neoantigens in Cancer. Front Oncol 2020;10:593989.

16. Bao BY, Pao JB, Huang CN, et al. Polymorphisms inside microRNAs and microRNA target sites predict clinical outcomes in prostate cancer patients receiving androgendeprivation therapy. Clin Cancer Res 2011;17:928-36.

17. Liu X, Song C, Yang S, et al. IFI30 expression is an independent unfavourable prognostic factor in glioma. J Cell Mol Med 2020;24:12433-43.

18. Love MI, Huber W, Anders S. Moderated estimation of fold change and dispersion for RNA-seq data with DESeq2. Genome Biol 2014;15:550.

19. Ito K, Murphy D. Application of ggplot2 to

Cite this article as: Jiang W, Zheng F, Yao T, Gong F, Zheng W, Yao N. IFI30 as a prognostic biomarker and correlation with immune infiltrates in glioma. Ann Transl Med 2021;9(22):1686. doi: 10.21037/atm-21-5569
Pharmacometric Graphics. CPT Pharmacometrics Syst Pharmacol 2013;2:e79.

20. Zhu M, Huang Y, Bender ME, et al. Evasion of innate immunity contributes to small cell lung cancer progression and metastasis. Cancer Res 2021;81:1813-26.

21. Walsh SR, Simovic B, Chen L, et al. Endogenous T cells prevent tumor immune escape following adoptive $T$ cell therapy. J Clin Invest 2019;129:5400-10.

22. Yu J, Qin B, Moyer AM, et al. Regulation of sister chromatid cohesion by nuclear PD-L1. Cell Res 2020;30:590-601.

23. Wang J, Sun J, Liu LN, et al. Siglec-15 as an immune suppressor and potential target for normalization cancer immunotherapy. Nat Med 2019;25:656-66.

24. Vago L, Gojo I. Immune escape and immunotherapy of acute myeloid leukemia. J Clin Invest 2020;130:1552-64.

25. Tan MSY, Sandanaraj E, Chong YK, et al. A STAT3based gene signature stratifies glioma patients for targeted therapy. Nat Commun 2019;10:3601.

26. Shi Y, Guryanova OA, Zhou W, et al. Ibrutinib inactivates BMX-STAT3 in glioma stem cells to impair malignant growth and radioresistance. Sci Transl Med 2018;10:eaah6816.

27. Wang Z, Shi Y, Ying C, et al. Hypoxia-induced PLOD1 overexpression contributes to the malignant phenotype of glioblastoma via NF- $\mathrm{KB}$ signaling. Oncogene 2021;40:1458-75.

28. Hoesel B, Schmid JA. The complexity of NF- $\kappa$ B signaling in inflammation and cancer. Mol Cancer 2013;12:86.

29. Ren D, Yang Q, Dai Y, et al. Oncogenic miR-210-3p promotes prostate cancer cell EMT and bone metastasis

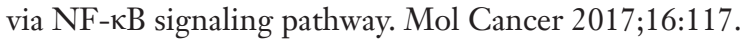

30. DiDonato JA, Mercurio F, Karin M. NF- $\kappa B$ and the link between inflammation and cancer. Immunol Rev 2012;246:379-400.

(English Language Editor: K. Brown) 